\title{
GPPS-BJ-2019-0179
}

\section{STUDY OF SLOPED TRENCH CASING TREATMENT ON PERFORMANCE AND STABILITY OF AXIAL COMPRESSORS}

\author{
HOU Jiexuan ${ }^{1}$, LIU Yangwei ${ }^{1,2, *}$ \\ 1. National Key Laboratory of Science and \\ Technology on Aero-Engine Aero- \\ Thermodynamics, School of Energy and Power \\ Engineering, Beihang University \\ 2 Collaborative Innovation Center of Advanced \\ Aero-Engine, Beihang University \\ Beijing, 100191, China \\ *Corresponding author: liuyangwei@126.com
}

Beijing, 100191, China

\begin{abstract}
A numerical simulation was carried out to investigate the effects of sloped trench casing treatment on performance and stability of axial compressors under both the isolated rotor and single stage environment. A detailed study of how the sloped trench casing treatment affects the flow structure, especially the tip leakage flow, and thus improves the performance of compressors is presented. Results show that the strength of the tip leakage vortex is weakened as the sloped trench casing treatment leads to less leakage flow entrained into it. Under the stage environment, the stability of the compressor is advanced as well as its performance since the downstream stator is working under a better inflow condition.
\end{abstract}

Keywords: compressor, casing treatment, sloped trench, tip leakage flow, numerical simulation

\section{INTRODUCTION}

A compressor is an important component of aero-engine and its design is a critical technology for the nation's industry. The complex vortex structure in the blade tip region which is an inherent flow phenomenon in the compressor has a great impact on compressor's aerodynamic loss and mass flow rate reduction. Furthermore, this phenomenon may lead to rotation stall and surge, so it is always a hot and difficult issue for international studies. In engineering application, some methods are used to control the tip region flow. And the casing treatment (Koch et al., 1970) is one which can improve the stability of the compressor with many advantages, such as simple structure, low cost, and high reliability (Robideau et al., 1980; Hemsworth et al., 1986). However, when the casing treatment works well in increasing the stall margin, it has problems with decreasing the total pressure ratio and efficiency at the same time (Greitzer et al., 1979; Fujita et al., 1984; Denton, 1993).

Wissler (1986) proposed a new type of casing treatment in his patent (structure shown in Figure 1). It is improved from rectangular groove casing treatment (Mohan et al., 1994; Thompson et al., 1998), by flattened the frontward step of the rectangular groove into a smooth inclined plane. The flow can be smoothly introduced into the mainstream and the loss thus be reduced. The experiment results (Wissler et al., 1987) show that, unlike the traditional casing treatment where the efficiency needs to be greatly sacrificed while expanding stability, the sloped trench casing treatment can take both efficiency and stability into account. The sloped trench casing treatment was then put into practical application. Zhu et al. (1998) carried out experiments on a single-stage subsonic axial compressor with different sloped trench casing treatment. The results show that the effect of sloped trench casing treatment is not constricted to the tip region, but alters the blade elementary features along the whole spanwise height. This conclusion is different from the result of Wissler et al. (1987) where the effect of the sloped trench casing treatment is considered within the tip region only. Zhang et al. (2007) numerically simulated the application of the sloped trench casing treatment in a transonic compressor rotor. The results show that the sloped trench casing treatment can reduce the entropy production in the rotor tip region, and thus increases the stall margin with slight loss of efficiency. This is contrary to the experimental results in subsonic compressor. Liu et al. (2014) performed numerical simulations on a transonic compressor rotor to study the sloped trench casing treatment with different structural parameters. The results show that with a relatively large tip clearance, the usage of the sloped trench casing treatment can improve the pressure ratio and the stall margin while the efficiency solely decreases near the stall point.

In the present available experimental and numerical studies, the effects of the sloped trench casing treatment on the stability and performance vary significantly with the working conditions. The illustrations of its mechanism are still not clear, and some research conclusions are even contradictory. 
In order to provide theoretical support for the engineering design, this paper introduces the sloped trench casing treatment to two different working environments, an isolated rotor case and a single stage case, to analyse its effects. The relationship among the trench casing treatment, the tip leakage flow and the compressor's performance and stability are also analysed to explain the mechanism of the sloped trench casing treatment.

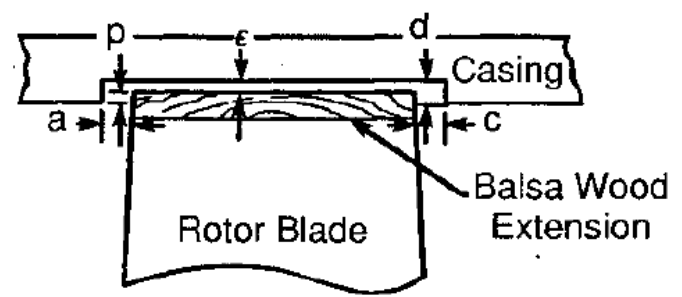

a) Cylindrical Trench Geometry

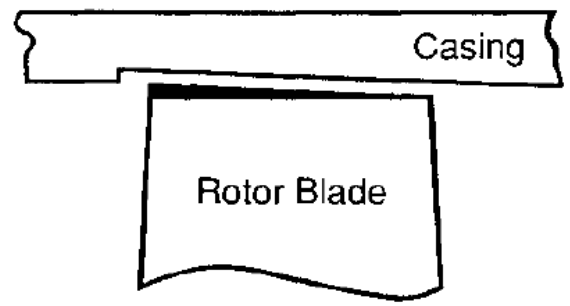

b) Sloped (Conical) Trench Geometry

\section{Figure 1 Sloped trench casing treatment configuration (Wissler et al., 1987)}

\section{METHODOLOGY}

The numerical simulation was conducted on Rotor4 (subsonic rotor) and Stage4 (subsonic stage) which are the parts of an actual compressor using sloped trench casing treatment. The parameters of the rotor and stator are showed in Table 1. The smooth casing cases were also simulated as the baselines by simply replacing the trench with a straight line. The configuration differences between smooth casing and trench casing are illustrated in Figure 2. The sloped trench casing treatment is applied to the rotor's shroud only.

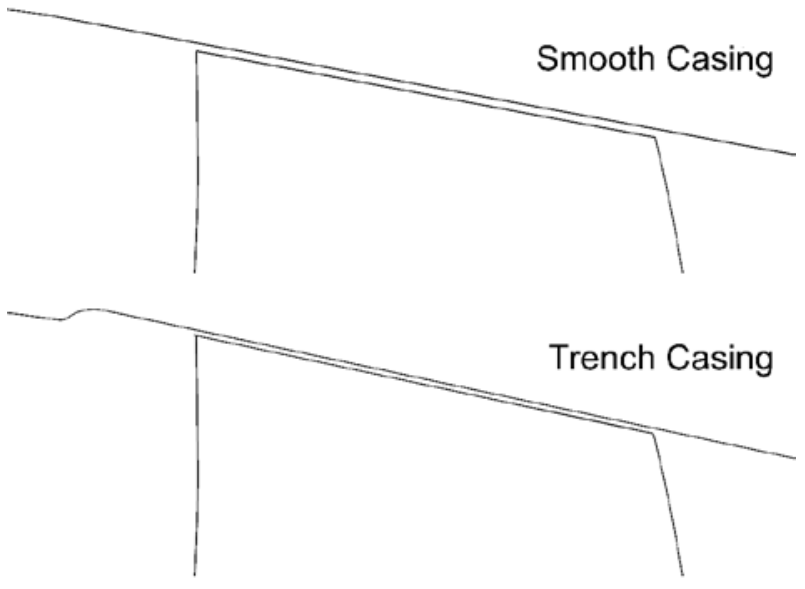

Figure 2 Configurations of smooth and trench casing

Table 1 Parameters of the rotor and stator

Rotating speed

Rotor clearance height

Trench depth

Trench step to blade

Rotor blade aspect ratio

Stator blade aspect ratio

Number of rotor blades

Number of stator blades

Reference Temperature

Reference pressure

$11994 \mathrm{rpm}$
$0.2 \mathrm{~mm}$
$0.4 \mathrm{~mm}$
$4 \mathrm{~mm}$
1.67
1.48
68
90
$288 \mathrm{k}$
$101325 \mathrm{pa}$

\section{Numerical Method}

The commercial software NUMECA is used for numerical study. Three-dimensional flow simulations were performed by solving the compressible Reynolds-averaged Navier-Stokes (RANS) equation. Jameson's central finite volume method with artificial viscosity is adopted for spatial discretization and Jameson's four-step Runge-Kutta method is adopted for time discretization. Spalart-Allmaras model, Standard K-Epsilon model and the SST K-Omega model are tested. All of the three models give the performance lines with a similar tendency. Considering the calculation efficiency and robustness, the turbulent viscosity was computed with the Spalart-Allmaras model in all the cases.

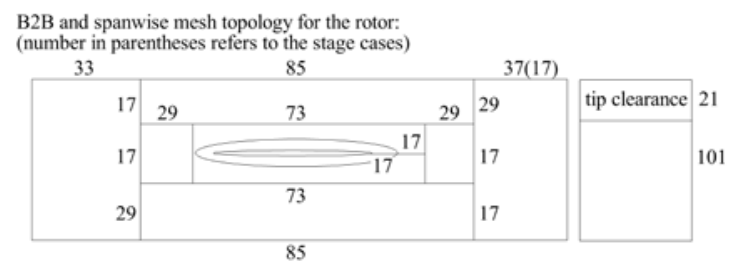

B2B and spanwise mesh topology for the stator:
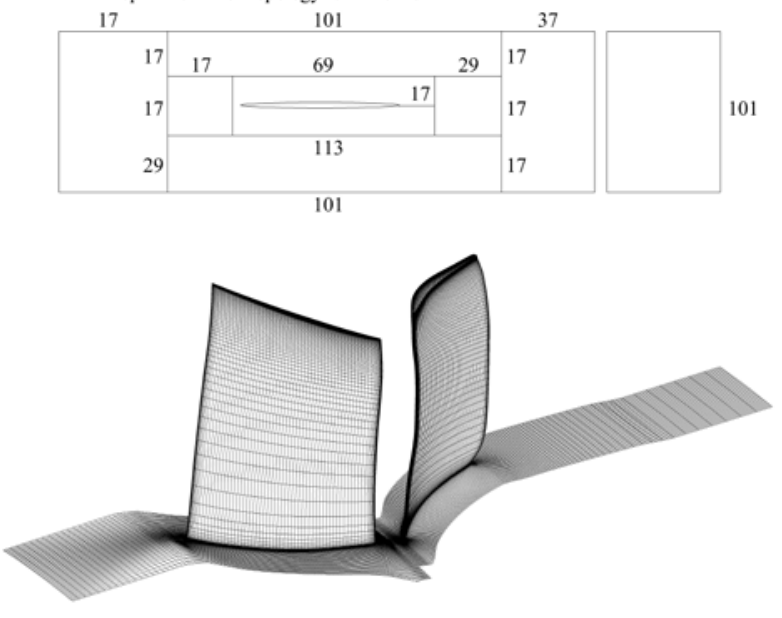

Figure 3 Mesh topology

Hexahedral structural meshes were generated by using NUMECA, Autogrid. One-blade passage was selected and periodic boundary conditions were set on the two sides of flow passage. A multi-block strategy was used to improve the grid quality, with $\mathrm{H}$-type, O4H-type, H-type and $\mathrm{OH}$-type grid topology applied to the inlet section, the blade section, the outlet section and the tip clearance section respectively for each blade row. The grids between each block were fully matched. To capture the flow structure, grid points are refined 
streamwise inside the sloped trench backward step and spanwise near the end-wall region. A series of meshes were generated with different distribution and grid densities to check the mesh independence and $y^{+}$availability for the simulation. After comparing the results of performance line computed from four different meshes, a mesh with 1.5 million grid points and $y^{+}$of first grid adjacent to the wall approximately 1.0 was picked for the further study. Detailed grid points assignment of the picked grid is showed in Figure 3. The mesh of the stage case was generated on the basis of the isolate rotor case with minimal change in the rotor region and adding the stator blade downstream. The total grid points of the stage case were 2.8 million. More information of the meshes see Hou (2017).

The profiles of total pressure, total temperature, and inlet flow angle were specified for the inlet boundary. At the outlet, profile of static pressure was prescribed based on the radial equilibrium equation. Nonslip and adiabatic conditions were adopted for all of the solid walls. In the stage case, the interface between two blade rows was set to the full nonmatching mixing plane.

\section{RESULTS AND DISCUSSION}

\section{Overall performance}

Figure 4 shows the performance lines of isolate rotor cases and the stage cases respectively. The steady-stable solution limit (the limit that the simulation cannot converge to a stable solution) is generally considered to be the stall point (Yamada et al., 2007; Vo et al., 2008). The x-axis value is nondimensionalized by the chock point mass flow rate of the smooth casing case.

Under the isolate rotor environment, the application of the sloped trench casing treatment doesn't affect the overall performance of the rotor significantly. Comparing with the smooth casing case, the absolute mass flow rate of the chock point increases by $1 \%$ which indicates that the flow capacity of the rotor is improved slightly; near the stall point, both the pressure ratio and the efficiency drops down a little; the stall margin is narrowed down slightly, as the comprehensive stall margin based on the peak efficiency (the calculating formula as below) decreases by $2.5 \%$.

$$
\begin{aligned}
& S M=\left(\frac{\dot{m}_{\text {peak }}}{\dot{m}_{\text {stall }}} \times \frac{\pi_{\text {stall }}}{\pi_{\text {peak }}}-1\right) \times 100 \% \\
& \Delta S M=S M_{\text {trench }}-S M_{\text {smooth }}
\end{aligned}
$$

Under the stage environment, the sloped trench casing treatment apparently improves the performance of the compressor. In the whole range of stable working, the pressure ratio in the trench casing case exceeds that in the smooth casing case. And the efficiency rises as well in a large scale except the working points near stall. Comparing to the smooth casing case, the absolute mass flow rate of the chock point increases by $1 \%$; the peak efficiency rises by $0.3 \%$, and the comprehensive stall margin extends by $0.45 \%$. The sloped trench casing treatment improves the performance of the compressor not only in stability but also in efficiency.
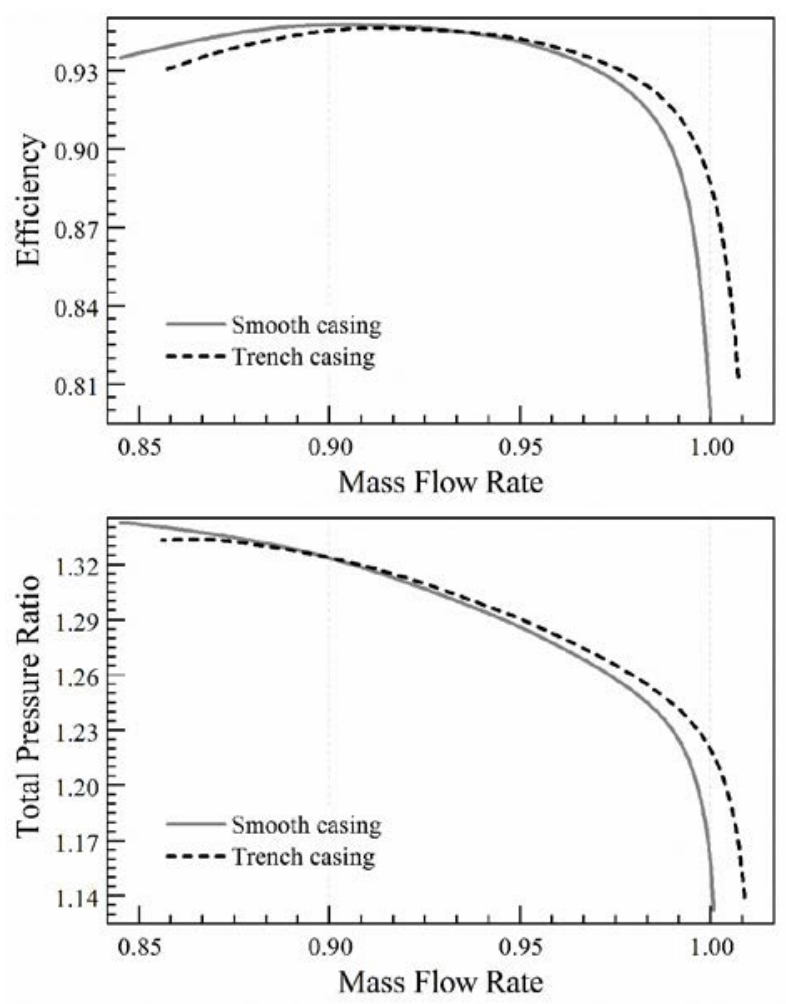

a) the isolate rotor cases
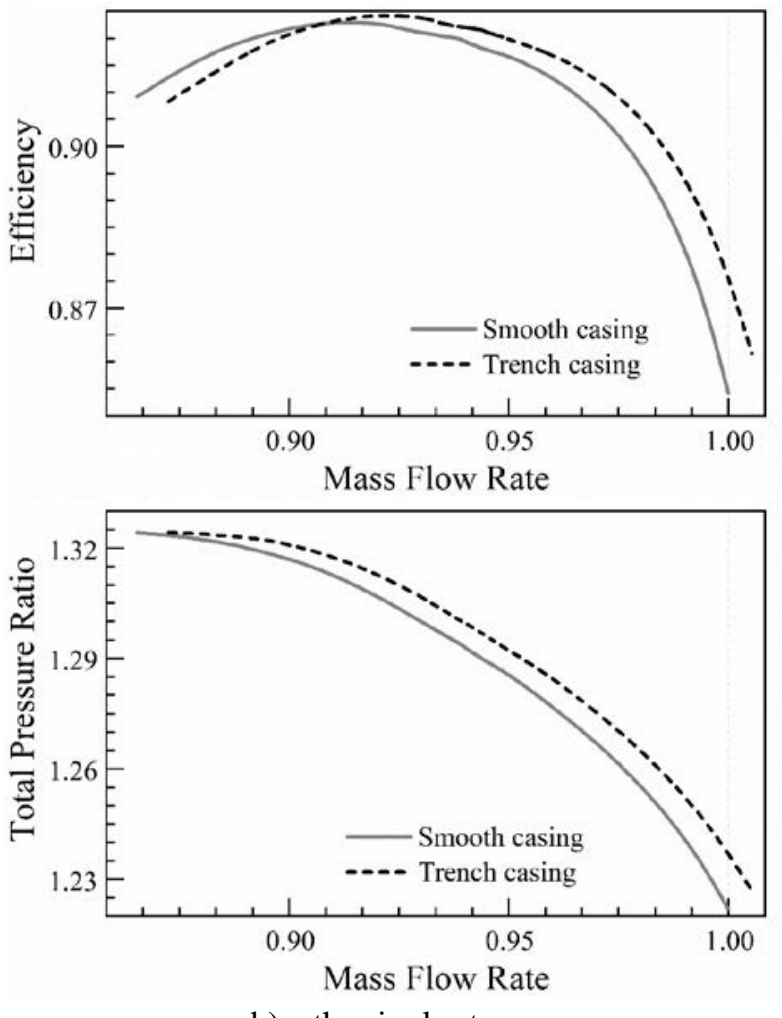

b) the single stage cases

Figure 4 Performance lines

To specify the effect of the sloped trench casing treatment on the flow field, working points near the chock points at 0.98 non-dimensional mass flow rate are chose for analysation 
under both isolate rotor environment and single stage environment. And for the stage cases, working points at 0.92 non-dimensional mass flow rate which is near the peak efficiency point are also picked for comparison.

\section{Near chock point}

Figure 5 shows the spanwise profile of efficiency (y axis value of spanwise coordinate is divided by the passage height) and the efficiency is calculated following the formula below:

$$
\eta_{a b}^{*}=\frac{\pi^{* \frac{k-1}{k}}-1}{\theta^{*}-1}\left(\pi^{*}=\frac{p_{2}^{*}}{p_{1}^{*}} ; \theta^{*}=\frac{T_{2}^{*}}{T_{1}^{*}} ; k=1.4\right)
$$

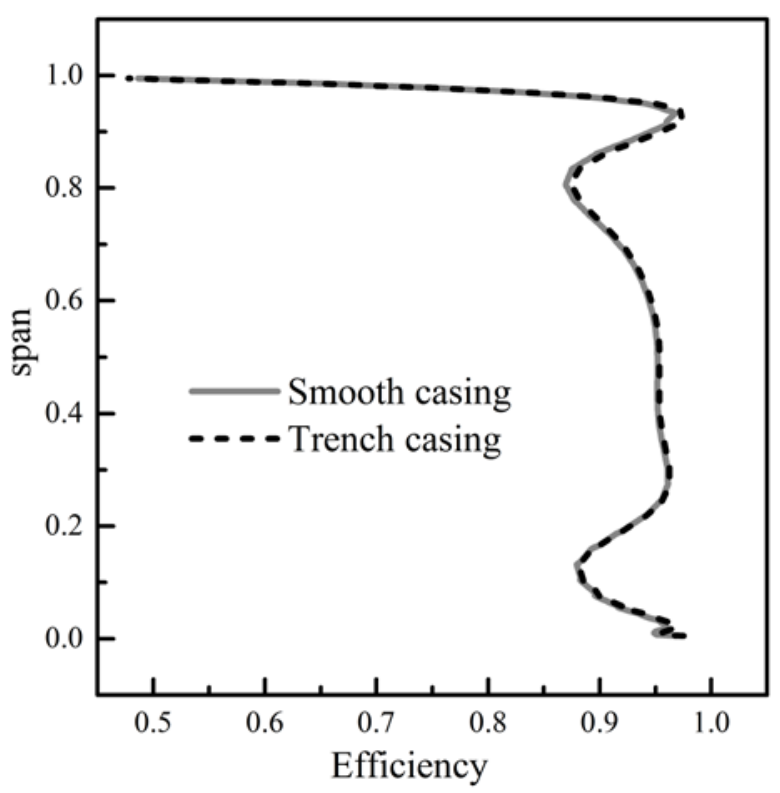

a) the isolate rotor cases

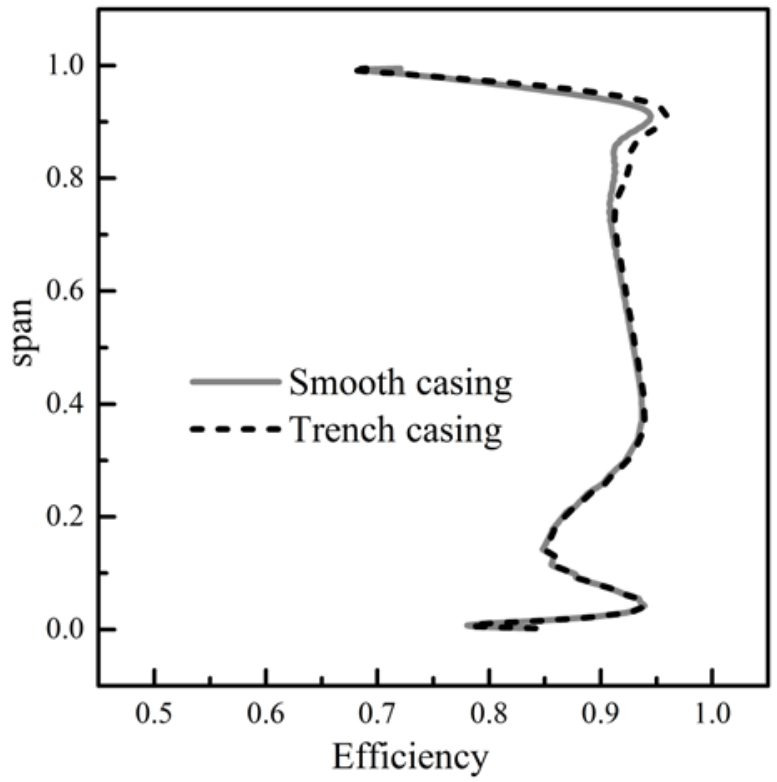

b) the single stage cases

Figure 5 Spanwise profile of efficiency

The subscript 1 refers to the inlet parameters, and the subscript 2 refers to the cross section at the axial position just downstream the stator's trailing edge. The flow parameters are mass weighted average at each spanwise height. Under the isolate rotor environment, the differences between two cases are relatively unobvious and restrict within the tip region where the efficiency of the trench casing case is greater than that of the smooth casing case at each same spanwise position. As to the stage cases, same changing tendency appears as the trench casing applied to the shroud, but the differences are more significant than that between the isolated rotor cases. The trench casing treatment rises the passage height, and the absolute mass flow rate of the chock point is thus increased.

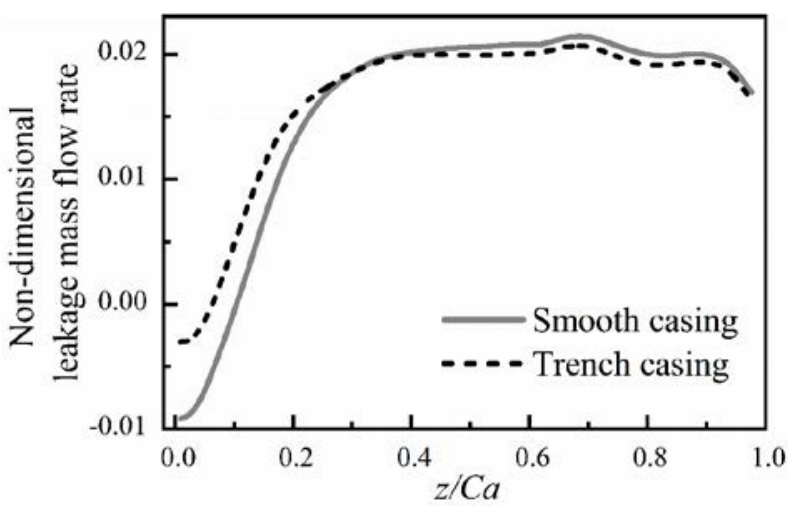

a) the isolate rotor cases

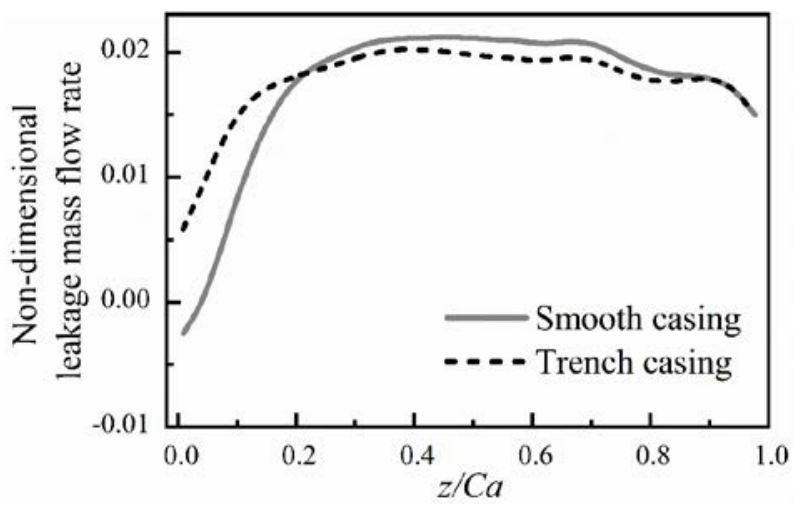

b) the single stage cases

\section{Figure 6 Non-dimensional leakage mass flow rate along the chord of the isolate rotor}

Figure 6 shows the leakage mass flow rate along the chord. The y-axis value of the leakage mass flow rate data is non-dimensionalized by the choke point mass flow rate of the smooth casing case. For both working environment, the absolute leakage mass flow rate in the trench casing case is larger than that in the smooth casing case. And the leakage flow can be divided into two parts based on the variation tendency. In the first part, the leakage mass flow rate gradually rises, and in the second part, the leakage mass flow rate remains at a stable level. Under the isolate rotor environment, the leakage mass flow rate reaches a similar stable level at a similar axial position in the second part. While under the single stage environment, the leakage flow comes into the second part at a more upstream position, and the value of the stable level is much lower in the trench casing case than that in the smooth casing case. Under both working environment, the leakage mass flow near the leading edge moves from negative towards positive after applying the trench casing 
treatment, which indicates that the attack angle increases. And the leakage mass flow rate in the smooth casing case climbs up in a shorter distance, which means a stronger leakage jet appears in the smooth casing case and leads to a stronger tip leakage vortex. The increase of the total leakage mass substantially comes from the first part, as in the second part the difference between two cases are much smaller. The effect of the trench casing treatment on the clearance leakage restricted to the fore part of the blade tip region.
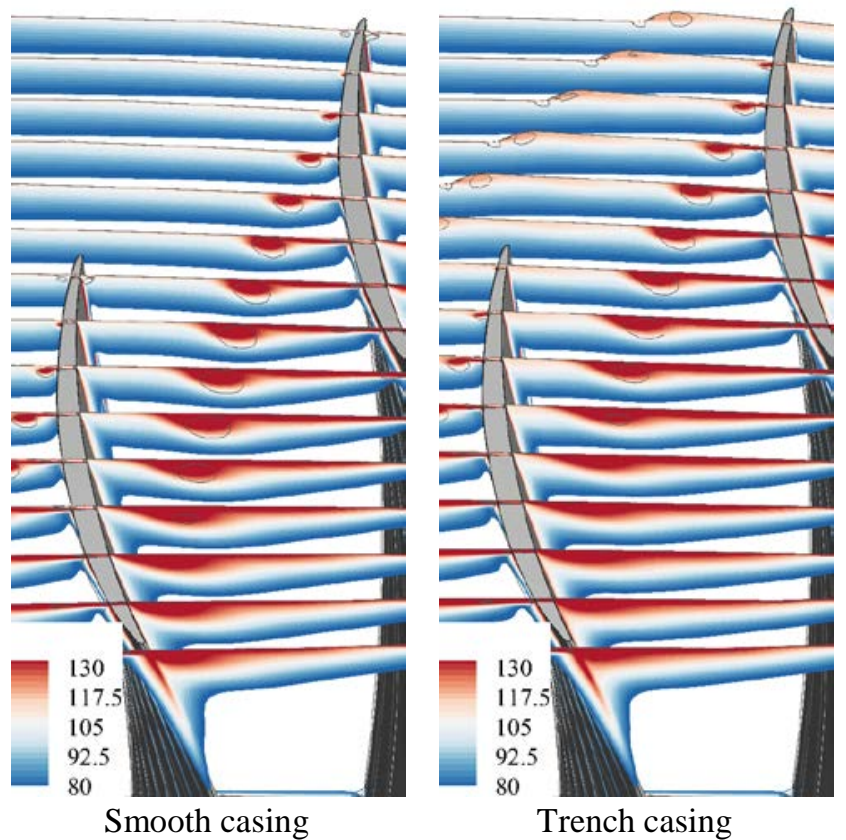

a) the isolate rotor cases

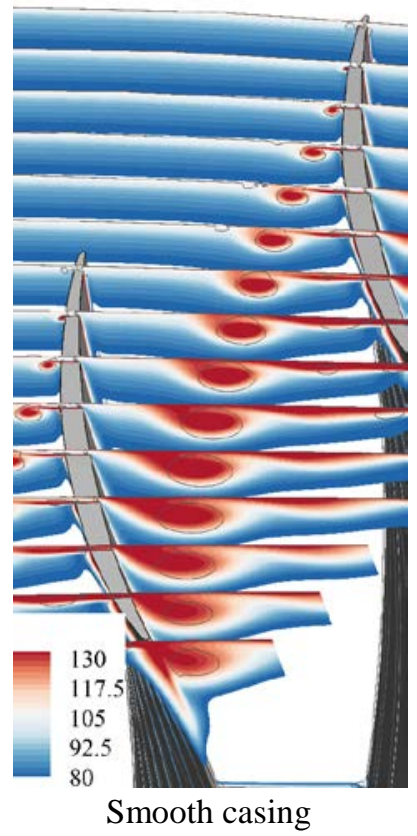

b) the single stage cases

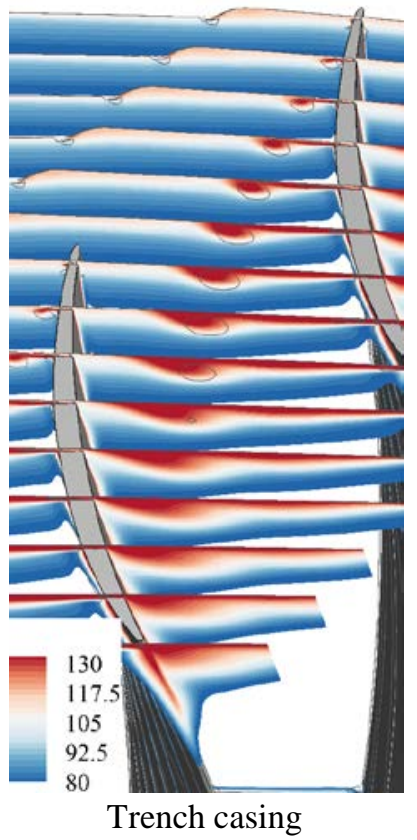

\section{Figure 7 Passage contours of entropy production}

In order to study the effects of trench casing treatment to the tip leakage flow structure, Figure 7 shows a zoomed in view of the flow field near tip region. The cross sections are perpendicular to the chord of the rotor tip. The contour map delineates the profile of entropy production (the calculating formula as below) and the grey lines are isolines of $Q=6 \times 10^{8}$. Here the Q criterion (Hunt et al., 1988) is used to represent the position of the tip leakage vortex.

$$
\mathrm{S}=\frac{c_{p}}{\gamma} \ln \left(\left(\frac{p}{p_{\text {ref }}}\right)^{1-\gamma} \cdot\left(\frac{T}{T_{\text {ref }}}\right)^{\gamma}\right)
$$

Under the isolate rotor environment, a vortex appears at the trench backward step region. This additional vortex accelerates the flow circumferentially near the tip leading edge and increases the attack angle. Comparing to the smooth casing case, the onset of the tip leakage vortex moves upstream; the high entropy production region inside the core zone of the tip leakage vortex diminishes and dissipates more quickly as the vortex transports downstream. The whole trajectory of the tip leakage vortex moves away towards the pressure side of the adjacent blade. More tip leakage flow is drawn into the clearance of the next blade as the second leakage flow, rather than flow out through the blade row exit. This flow structure results in a reduction in the passage blockage and an improvement in the efficiency. When it comes to the stage environment, the weakening of the tip leakage vortex by the trench casing is more pronounced. The max value of the high entropy production region inside the vortex core zone decreases from its initial rolling up and dissipates much quickly to the downstream, while the tip leakage vortex still remains a rounded form at the passage exit in the smooth casing case. Since the primary cause of the flow field nonuniform diminishes, the stator blades are thus working under a better inlet condition and its performance improves.

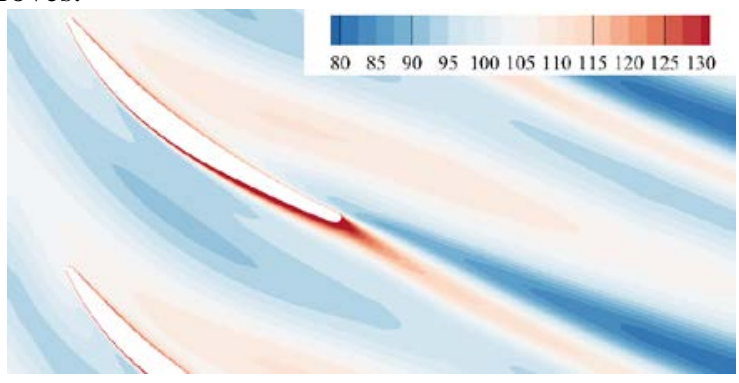

a) the isolate rotor cases

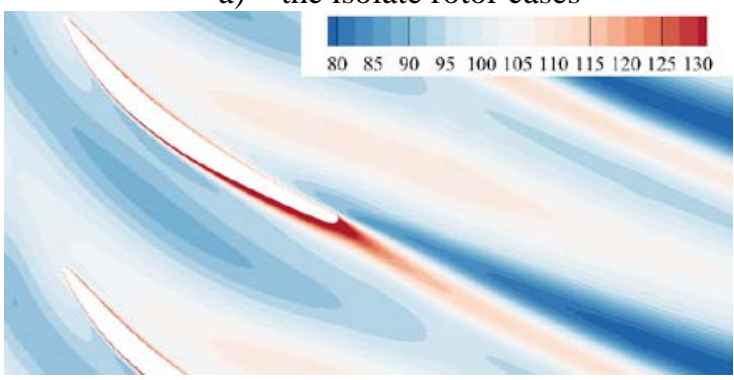

b) the single stage cases

\section{Figure 8 Entropy contour at $98 \%$ spanwise height of stator}

The above analyses show that the trench casing treatment cannot work well solely with an isolate blade, and it gains benefits mainly from the following stators which are working at a better environment provide by the upstream rotors. Figure 8 shows the contour of entropy at $98 \%$ spanwise height of 
stator. The left-most position of the figure refers to the interface of two rows, where the entropy of the trench casing case is obviously lower than that of the smooth casing case. Inside the stator passage, the high entropy zone due to the upstream tip leakage vortex is cut down by the trench casing. The mass weighted average entropy production at the cross section upstream the stator leading edge reduces 5\% after introducing the trench casing treatment to the shroud. Figure 11 shows the spanwise profile of the entropy production difference between the stator inlet and outlet. For the 20\% spanwise height region near the outer casing, the entropy production of the trench casing case is much smaller than that of the smooth casing case. The benefits of the trench casing treatment further magnify in the stator flow.

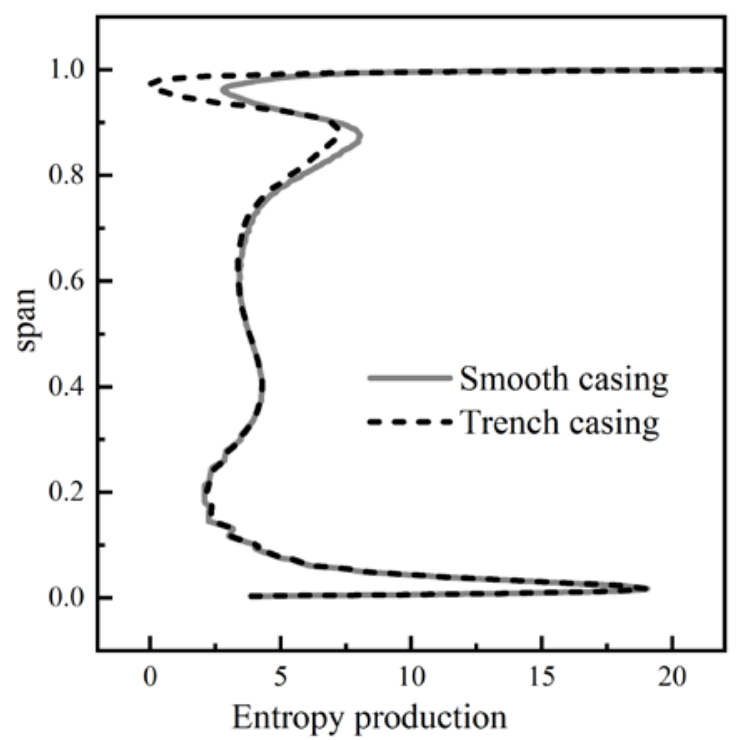

\section{Figure 9 Spanwise profile of the entropy production difference between stator inlet and outlet}

\section{Near peak efficiency point}

Figure 10 shows the spanwise profile of efficiency near the peak efficiency point. In the near shroud region, the loss brought in by the trench appears more obvious than that of the near chock point cases. In the near tip region, where the tip leakage flow domains the flow field, the efficiency of the trench casing case remains higher than that of the smooth casing case at each same spanwise height. The capability of the trench casing treatment suppressing the tip leakage vortex still plays a role. The general effects of the trench casing treatment stay the same with that of the near chock point cases.

Figure 11 shows the contours of entropy production of cross sections which are perpendicular to the chord of the rotor tip. At this working point, the circumferential movement of the inflow is much stronger than that in the chock point cases, and the attack angle becomes larger and positive. As a result, the influence on the acceleration of the flow in the tip region near the leading edge by the circumferential vortex at the trench region is not as obvious as that in the near chock point. As the tip leakage vortex transports more crosswise to the pressure side of the next blade, the onset of the second leakage flow moves upstream at the fore part of the blade tip. This leads to the leakage flow which rolls up to form the initial tip leakage vortex holds a large entropy production before entering the blade clearance and the high entropy production region inside the tip leakage vortex zone is thus much larger than that in the smooth casing case. However, as the tip leakage flow drawn into the second leakage flow, the tip leakage flow is then weakened, and the blockage caused by the tip leakage vortex is also reduced. The trench casing treatment still acts as a positive method while the stability of the stage is not significantly affected. The main effect of the trench casing treatment is to improve the performance of the compressor, and the main reason that the stall margin is slightly improved is the mass flow rate at the near chock point increases.

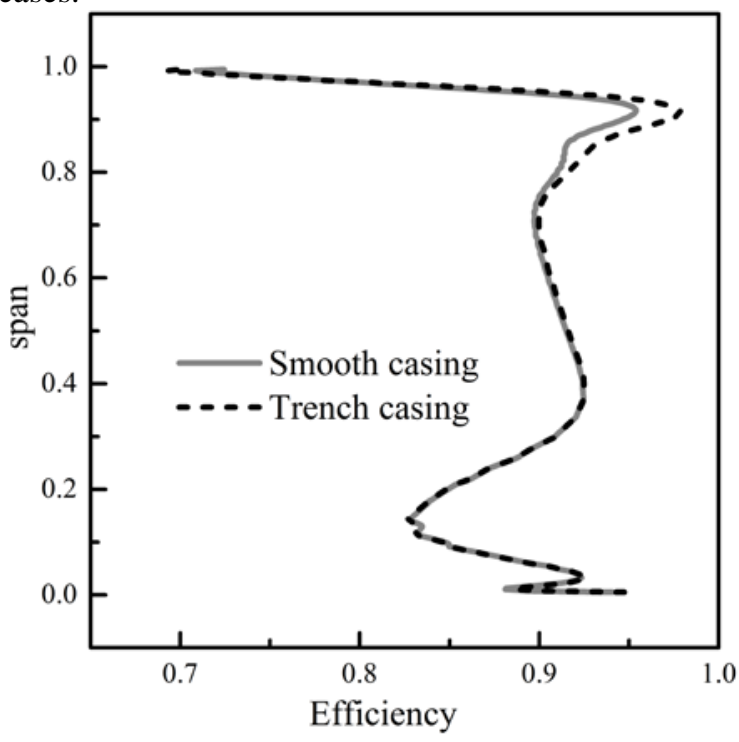

Figure 10 Spanwise profile of efficiency

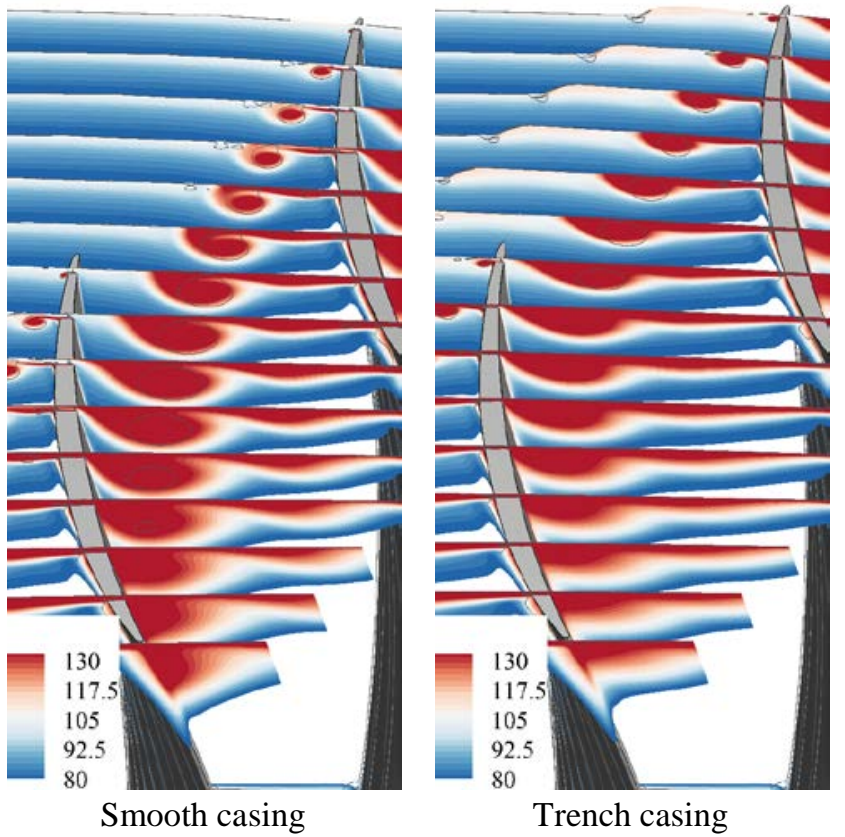

Figure 11 Passage contours of entropy production at near peak efficiency point 


\section{CONCLUSIONS}

The significant conclusions of this study are as follows:

1. When the sloped casing treatment is applied to a rotor, the flow near the trench is then circumferentially accelerated, and the attack angle is thus increased.

2. The tip leakage vortex is weakened and the blockage inside the flow passage is reduced by the trench casing treatment. The improvement of the flow in the tip region reduces the loss and increases the performance of the rotor to some degree.

3. With a isolate rotor, the sloped trench casing treatment doesn't work well. However, under the stage environment, both the efficiency and pressure ratio are improved. The stability slightly improved, since the mass flow rate at chock point increases while that at stall point drops little.

4. The benefit of the sloped trench casing treatment expands under the stage environment. The design of the trench casing should take the stage environment as an integral.

\section{ACKNOWLEDGMENTS}

This work is supported by the National Natural Science Foundation of China (No. 51676007, No. 51420105008, No. 51790513). We express our appreciation to the late Prof. Lipeng Lu, whose contribution to this work was of great significance.

\section{References}

Denton, J. D. (1993). Loss mechanisms in turbomachines. ASME 1993 International Gas Turbine and Aeroengine Congress and Exposition (pp. V002T14A001V002T14A001). American Society of Mechanical Engineers. 10.1115/93-GT-435

Fujita, H., Takata, H. (1984). A study on configurations of casing treatment for axial flow compressors. Bulletin of JSME, 27(230), 1675-1681. 10.1299/jsme1958.27.1675

Greitzer, E. M., Nikkanen, J. P., Haddad, D. E., Mazzawy, R. S., Joslyn, H. D. (1979). A fundamental criterion for the application of rotor casing treatment. Journal of Fluids Engineering, 101(2), 237-243. 10.1115/1.3448945

Hemsworth, M. C. (1986). U.S. Patent No. 4,606,699. Washington, DC: U.S. Patent and Trademark Office.

Hou J. (2017). Study of sloped trench casing treatment on preformance of axial compressures. Master Dissertation. Beihang University.

Hunt, J. C., Wray, A. A., Moin, P. (1988). Eddies, streams, and convergence zones in turbulent flows. Studying Turbulence Using Numerical Simulation Databases, 2. Proceedings of the 1988 Summer Program; p. p 193-208

Koch, C. C., Smith L H. (1970). Experimental evaluation of outer case blowing or bleeding of single stage axial flow compressor, part 6 Final report. NASA CR-54592.

Liu S., Ning F. (2014). Numerical investigation of the effects of sloped trench casing treatment on the aerodynamic performance of axial compressor. Gas Turbine Experiment and Research. 2014(5):5-11.

Mohan, K., Guruprasad, S. A. (1994). Effect of axially non-uniform rotor tip clearance on the performance of a high speed axial flow compressor stage. ASME 1994 International Gas Turbine and Aeroengine Congress and Exposition (pp. V001T01A150-V001T01A150). American Society of Mechanical Engineers. 10.1115/94-GT-479

Robideau, B. A., Niiler, J. (1980). U.S. Patent No. 4,238,170. Washington, DC: U.S. Patent and Trademark Office.

Thompson, D. W., King, P. I., Hah, C., Rabe, D. C. (1998). Experimental and computational investigation of stepped tip gap effects on the flowfield of a transonic axialflow compressor rotor. ASME 1998 International Gas Turbine and Aeroengine Congress and Exhibition (pp. V001T01A026V001T01A026). American Society of Mechanical Engineers.10.1115/94-GT-479

Vo, H. D., Tan, C. S., Greitzer, E. M. (2008). Criteria for spike initiated rotating stall. Journal of turbomachinery, 130(1), 011023. 10.1115/1.2750674

Wisler, D. C. (1987). U.S. Patent No. 4,645,417. Washington, DC: U.S. Patent and Trademark Office.

Wisler, D., \& BEACHER, B. (1986). Improved compressor performance using recessed clearance (trenches) over the rotor. 22nd Joint Propulsion Conference (p. 1745). 10.2514/6.1986-1745

Yamada, K., Funazaki, K., Furukawa, M. (2007). The behavior of tip clearance flow at near-stall condition in a transonic axial compressor rotor. ASME turbo expo 2007: Power for land, sea, and air (pp. 295-306). American Society of Mechanical Engineers. 10.1115/GT2007-27725

Zhang, H., Ma, H. (2007). Study of sloped trench casing treatment on performance and stability of a transonic axial compressor. ASME Turbo Expo 2007: Power for Land, Sea, and Air (pp. 399-404). American Society of Mechanical Engineers. 10.1115/GT2007-28140

Zhu j., Zhao Y., Liu Z. (1998). An experimental investigation on treatment of compressor casing with skew grooves. Journal of Aerospace Power(1), 23-26. 